\title{
Technical Sentiment Analysis: Measuring Advantages and Drawbacks of New Products Using Social Media
}

\section{Chiarello, Filippo $^{a}$; Bonaccorsi, Andrea ${ }^{a}$; Fantoni, Gualtiero ${ }^{\text {;OSssola, Giacomo }}{ }^{\text {; }}$; Cimino, Andrea ${ }^{b}$ and Dell'Orletta, Felice ${ }^{b}$}

${ }^{a}$ Department of Energy, Systems, Territory, and Construction Engineering, University of Pisa, Italy. ${ }^{\mathrm{b}}$ Institute for Computational Linguistics of the Italian National Research Council (ILC- CNR)

\begin{abstract}
In recent years, social media have become ubiquitous and important for social networking and content sharing. Moreover, the content generated by these websites remains largely untapped. Some researchers proved that social media have been a valuable source to predict the future outcomes of some events such as box-office movie revenues or political elections. Social media are also used by companies to measure the sentiment of customers about their brand and products.
\end{abstract}

This work proposes a new social media based model to measure how users perceive new products from a technical point of view. This model relies on the analysis of advantages and drawbacks of products, which are both important aspects evaluated by consumers during the buying decision process. This model is based on a lexicon developed in a related work (Chiarello et. al, 2017) to analyse patents and detect advantages and drawbacks connected to a certain technology.

The results show that when a product has a certain technological complexity and fuels a more technical debate, advantages and drawbacks analysis is more efficient than sentiment analysis in producing technical-functional judgements.

Keywords: Social media; Twitter; Sentiment analysis; Product Success 


\section{Introduction}

Nowadays, social media have become an inseparable part of modern life, providing a vast record of mankind's everyday thoughts, feelings and actions. For this reason, there has been an increasing interest in research of exploiting social media as information source of knowledge although extracting a valuable signal is not a trivial task since social media data is noisy and must be filtered before proceeding with the analysis. In this domain, sentiment analysis, which aims to determine the sentiment content of a text unit, is considered one of the best data mining method. It relies on different approaches (Collomb et al. 2013) and it has been used to answer research questions in a variety of fields comprised the measure of customers perception of new products (Mirtalaie et al. 2018).

In this work, we try to understand if sentiment analysis is really the best available method to analyse consumer's perception of products, expecialy when we want to measure the perception of the technical content of the product. Thus we compare State of the art sentiment analysis techniques with a lexicon of advantages and drawbacks related to products. This tool relies on a lexicon developed by Chiarello (2017) to extract advantages and drawbacks of inventions from patents.

Our work started with the selection of an event able to polarise Twitter users' attention and products to analyse. In particular, we chose a premiere tradeshow for the video game industry, and two video game consoles disclosed during the event. We collected about 7 milions tweets about products published before, during and after the tradeshow. Since social media data is noisy (for example it may contains spam and advertising), before proceeding with the analyses, we filtered our dataset. In particular, after removing too short and non-English tweets, we manually classified a randomly extracted subset of posts to train a classifier which provide us the cleansed dataset. Then we conducted a sentiment analysis of the tweets using state of the art machine learning techniques. We classified each tweet as positive, negative or neutral. At this point we applied our lexicon identifying advantages tweets and drawbacks tweets. Finally we compared the outputs of the two analyses for the two product-related clusters of tweets.

We found consistent differences between the extractions. The results shows that when a product has a certain technological complexity and fuels a more technical debate, advantages and drawbacks analysis is more able than sentiment in producing technicalfunctional judgements. For this reason we think that the proposed methodology peforms better then standard sentiment analysis techniques when a product has a certain technological complexity and fuels a more technical social media discourse. 


\section{State of the art}

We provide an overview of the studies about social media forecasting (Table 1,2). Researchers especially focused on economics (stock market, marketing, sales) and politics (elections outcomes). In economics, predicting fluctuations in the stock market has been the most studied by far. Early work focused largely on predicting whether aggregate stock measures such as the Dow Jones Industrial Average (DJIA) would rise or fall on the next day, but forecasting can also involve making more detailed predictions, e.g., forecasting market returns or making predictions for individual stocks. The simplest task for stock market prediction is deciding whether the following day will see a rise or fall in stock prices. Comparison between studies is complicated by the fact that stock market volatility, and thereby the difficulty of prediction, may vary over time periods. High accuracy $(87,6 \%)$ on this task was reported by Bollen (2012). However, slight deviations away from their methodology have seen much less success indicating that the method itself may be unreliable (Xu, 2014). A very good result is achieved by Cakra (2015) who use linear regression to build a prediction model based on the output of sentiment analysis and previous stock price dataset.

Social media has also been used to study the ability of online projects to successfully crowdfund their projects through websites like Kickstarter. Li (2016) predicts whether a project will eventually succeed by making use of features relevant to the project itself (e.g., the fundraising goal), as well as social activity features (e.g., number of tweets related to the project), and social graph measures (e.g., average number of followers for project promoters). Using all of these features for only the first $5 \%$ of the project duration achieved an AUC of 0.90 , reflecting very high classification performance.

Many studies analysed the predictive power of social media to improve or replace traditional and expensive polling methods. The simplest technique is measuring tweet volume (tweet mentioning a political party $=$ votes). Chung (2010) and Tumasjan (2010) employed this method obtaining mixed results. Razzaq (2014), Skoric (2012) and Prasetyo (2015) improved this method taking into account the mood of the posts, considering if a candidate or a party is mentioned in a positive or negative manner. 
Table 1: Summary of studies in economics. Data source: $T=$ Twitter, $F=$ Facebook, $\mathrm{K}=$ Kickstarter, $\mathrm{O}=$ blogs, other. Task: MDA = Mean Directional Accuracy, MAPE = Mean Absolute Percentage Error.

\begin{tabular}{|c|c|c|c|c|c|}
\hline Article & Topic & Data source & Data size & Observation time & Success rate \\
\hline Xu (2014) & Stock market & $\mathrm{T}$ & $100 \mathrm{~K}$ tweets & 42 days & $M D A=58.9 \%$ \\
\hline Crone (2014) & Exchange rates & $\mathrm{T}, \mathrm{F}, \mathrm{O}$ & N/A & N/A & $M D A=60.26 \%$ \\
\hline Kordonis (2016) & Stock market & $\mathrm{T}$ & N/A & N/A & $M D A=87 \%$ \\
\hline Cakra (2015) & Stock market & $\mathrm{T}$ & N/A & 2 weeks & $R^{2}=0.9983$ \\
\hline Bollen (2015) & Stock market & $\mathrm{T}$ & $9.8 \mathrm{M}$ & 10 months & $M D A=87.6 \%$ \\
\hline Brow (2012) & Stock market & $\mathrm{T}$ & $13 \mathrm{~K}$ & 9 days & $r=0.62$ \\
\hline Rao (2012) & Stock market & $\mathrm{T}$ & $4 \mathrm{M}$ & 14 months & $\begin{array}{c}R^{2}=0.95 \text { (DJIA); } \\
R^{2}=0.68 \text { (NASDAQ) }\end{array}$ \\
\hline Kim (2014) & Hit songs & $\mathrm{T}$ & $31.6 \mathrm{M}$ & 68 days & $F 1=0.841$ \\
\hline Korolov (2015) & Donations & $\mathrm{T}$ & $15 \mathrm{M}$ & 10 days & $R^{2}=0.9286$ \\
\hline Le (2015) & Sports book & $\mathrm{T}$ & $1.2 \mathrm{M}$ & 30 days & $\begin{array}{l}A U C=80 \% \\
\text { Return }=8 \%\end{array}$ \\
\hline Tuarob (2013) & Smartphone sales & $\mathrm{T}$ & $800 \mathrm{M}$ & 19 months & $r=0.8837$ \\
\hline Asur (2010) & Movie revenues & $\mathrm{T}$ & $2.8 \mathrm{M}$ & 3 months & $R^{2} a d j=0.94$ \\
\hline Ahn (2014) & Car sales & $\mathrm{T}, \mathrm{F}, \mathrm{O}$ & $26 \mathrm{~K}$ posts & N/A & $\begin{array}{c}R M S E=0.170 \\
(\text { Sedan A); } \\
R M S E=0.232 \\
(\text { Sedan B })\end{array}$ \\
\hline Chen (2015) & Advertising & $\mathrm{T}$ & $5.9 \mathrm{~K}$ users & N/A & $\begin{array}{l}66 \% \text { gain (click rate); } \\
87 \% \text { gain (follow rate). }\end{array}$ \\
\hline Li (2016) & $\begin{array}{l}\text { Crowdfunding success } \\
\text { rate }\end{array}$ & $\mathrm{T}, \mathrm{F}, \mathrm{K}$ & $106 \mathrm{~K}$ tweets & 6 months & $A U C=0.90$ \\
\hline
\end{tabular}

Researchers employ different tools and methods for social media mining, varying from easy to somewhat more complex. The most employed tool is sentiment analysis (with its various approaches: knowledge-based techniques, statistical methods, and hybrid approach) which usually achieves good results. Other researchers use more complex tools such as neural networks or a combination of techniques. At end of the analysis of the state of the art we are able to identify some best practices: (i) implementing suitable techniques to deal with noisy data, (ii) evaluating statistical biases in social media data, (iii) collecting data from heterogeneous sources, (iv) incorporating domain-specific knowledge to improve statistical model. 
Table 2: Summary of studies in politics. Data source: $\mathbf{T}=$ Twitter. Task: Acc. $=$ Accuracy, MDA = Mean Directional Accuracy, MAPE = Mean Absolute Percentage Error .

\begin{tabular}{|c|c|c|c|c|c|}
\hline Article & Topic & Data source & Data size & Observation time & Success rate \\
\hline Chung (2010) & Renewal of US senate & $\mathrm{T}$ & $235 \mathrm{~K}$ tweets & Acc. $41 \%-47 \%$ \\
\hline Tumasjan (2010) & German federal election & $\mathrm{T}$ & $104 \mathrm{~K}$ tweets & N/A days \\
\hline Razzaq (2014) & Pakistani election & $\mathrm{T}$ & $613 \mathrm{~K}$ tweets & Acc. 50\% $1.65 \%$ \\
\hline Skoric (2012) & Political election & $\mathrm{T}$ & $7 \mathrm{M}$ tweets & 36 days & MAE 6.1\% \\
\hline Prasetyo (2015) & Indonesian political election & $\mathrm{T}$ & $7 \mathrm{M}$ tweets & 83 days \\
\hline
\end{tabular}

\section{Methodology}

\subsection{Selection of a triggering event and products}

We chose the Electronic Entertainment Expo as event able to polarise users' attention. Commonly referred to as E3, it is a premier trade event for the video game industry, presented by the Entertainment Software Association (ESA). We chose two new video game consoles, disclosed at E3 2017, as products of which predicting the success or failure. The first is Xbox One X, a new high-end version of Xbox One with upgraded hardware and the other product is New Nintendo 2DS XL, a streamlined version of the handheld console New Nintendo 3DS XL.

\subsection{Data collection}

Twitter provides two possible ways to gather tweets: the Streaming Application Programming Interface (API) and the Search API. The first one allows user to obtain realtime access to tweets from an input query. The user first requests a connection to a stream of tweets from the server. Then, the server opens a streaming connections and tweets are streamed in as they occur, to the user. However, there are a few limitations of the Streaming API. First, language is not specifiable, resulting in a stream that contains tweets of all languages, including a few non-Latin-based alphabets, that complicates further analysis. Instead, Twitter Search API is a Representational State Transfer API which allows users to request specific queries of recent tweets. It allows filtering based on language, region, geolocation, and time. Unfortunately, using the Search API is expensive and there is a rate limit associated with the query. Because of these issues, we decided to go with the Twitter Streaming API instead. For each product, we detected related hashtags and keywords an constructed a query to download relevant tweets.

We chose to collect tweets not only after the tradeshow, but also before. For these reason, we initially identified some products keywords with their provisional names and we updated them at a later stage. Tweets have been downloaded from CNR (Consiglio 
Nazionale delle Ricerche, Istituto di Informatica e Telematica, Area di Pisa) since 11th June 2017 h. 10:00 to 31th July 2017 h. 15:00.

\subsection{Data filtering}

The initial dataset resulted to be very noisy, containing tweets written in different languages, advertising and posts related to different products or subjects. We chose to keep into account only English tweets because sentiment and advantages/drawbacks lexicon is in this language. The data set is filtered removing tweets with less than five words and nonEnglish posts with a language classifier. We obtained 7.165.216 of filtered tweets.

At this point we created a golden set of relevant tweet to train a Supported Vector Machine classifier able to recognize relevant and unrelevant tweets. We defined characteristics that make a tweet: (i) relevant (posted by users or containing words or opinions related to our products of interests and their functionalities), (ii) irrelevant (tweets containing advertisings, links to e-commerce websites or messages related to other products or subjects). A researcher manually classified a subset made up of randomly extracted tweets. In particular, we exctract a subset composed of 6.500 finding 105 positive results and 6.395 negative. SVM model was then trained using this dataset, and computed a probability for each tweet to be relevant or irrelevant. A threshold of 0.7 has been chosen to label a tweet as relevant. The final dataset of filtered tweets, made up of 66.796 posts. We clustered tweets using product-related keywords. Clustering posts allowed us to further filter the final dataset which contained a small number of irrelevant tweets (Table 3).

Table 3. Clusters of tweets

\begin{tabular}{ccc}
\hline & $\mathbf{N}^{\circ}$ of tweets & \% of tweets \\
\hline Xbox One X & 64.885 & $97,14 \%$ \\
$\begin{array}{c}\text { New N2DS } \\
\text { Irrelevant } \\
\text { tweets }\end{array}$ & 1.706 & $2,55 \%$ \\
\hline
\end{tabular}

Table 4. Sentiment analysis classification

\begin{tabular}{cccc}
\hline & Positive & Negative & Neutral \\
\hline Xbox One X & $35,99 \%$ & $4,65 \%$ & $59,37 \%$ \\
New N2DS & $52,99 \%$ & $1,58 \%$ & $45,43 \%$ \\
Overall & $36,42 \%$ & $4,57 \%$ & $59,01 \%$ \\
\hline
\end{tabular}

\subsection{Sentiment analysis}

Table 4 presents the results of the sentiment analysis. We classified each tweet according to its sentiment into positive, negative, or neutral. We used an established methodology 
developed by Cimino (2016). We pre-processed the tweets by removing mentions (@ character), URLs, product hashtags, emoticons and single characters. As a result, for each tweet we obtained a probability of belonging to a mood class. After a manual analysis, we used a class prediction probability threshold of 0.6 to filter out low confidence prediction, i.e. tweets that cannot be classified as positive or negative with a high confidence are classified as neutral instead.

\subsection{Advantages and drawbacks analysis}

To extract technical advantages and drawbacks from tweets we used the lexicon developed in Chiarello (2017) that contains 657 Advantages words and 297 Drawbacks clues. These words are searched on our dataset finding different percentages of tweets with words from the lexicon in the two product-related clusters of tweets. Table 5 reports the results.

Table 5: Percentages of tweets containing or not words from our lexicon.

\begin{tabular}{cccccc}
\hline & $\begin{array}{c}\text { Tweets with } \\
\text { adv }\end{array}$ & $\begin{array}{c}\text { Tweets with } \\
\text { drw }\end{array}$ & $\begin{array}{c}\text { Tweets with } \\
\text { adv \& drw }\end{array}$ & $\begin{array}{c}\text { Tweets with } \\
\text { no adv or drw }\end{array}$ & $\begin{array}{c}\text { Tweets with } \\
\text { adv or drw }\end{array}$ \\
\hline Xbox One X & $8,84 \%$ & $3,74 \%$ & $0,37 \%$ & $87,05 \%$ & $12,95 \%$ \\
New N2DS XL & $6,62 \%$ & $0,94 \%$ & $0,00 \%$ & $92,44 \%$ & $7,56 \%$ \\
\hline
\end{tabular}

\section{Results: Comparison Between Sentiment Analysis and Technical Advantages and Disadvantage Extraction}

We adapted the advantages \& drawbacks analysis to give as output a classification ef each tweet. We classified data coming from the latter analysis in this way: (i) positive (tweets containing only advantages words), (ii) negative (tweets containing only drawbacks), (iii) neutral (tweets with no words of our lexicon or controversial tweets). As we can see in figure 2, sentiment analysis is more able to polarise tweets. In fact, with this analysis we found lower levels of neutral tweets, respectively $59.37 \%$ for Xbox One X and $45.43 \%$ for the New Nintendo 2DS XL. 


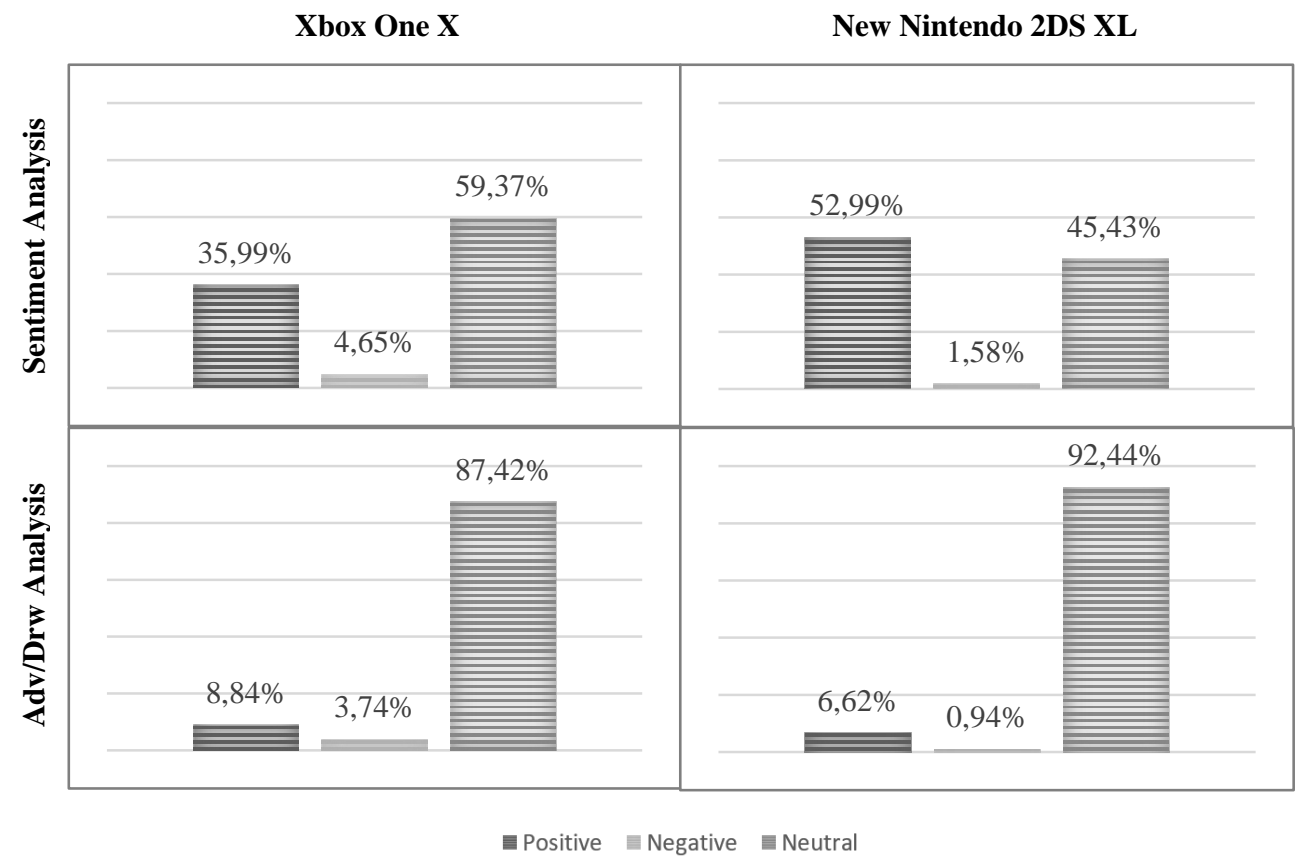

Figure 1: Comparison between Sentiment analysis and Advantages/Drawbacks analysis

This was an expected result since this kind of analysis is designed to deal with colloquial language while our lexicon is technical, being derived from patents analysis. What surprised us is the different polarisation of the products that we see comparing the two analyses. In fact, while with sentiment analysis Nintendo achieves lower percentages of neutral tweets, with advantages and drawbacks analysis is the opposite, since Xbox tweets are more polarised. We also noted that we found more tweets with words of our lexicon in the Xbox subset than in the Nintendo one (Table 5). We did the hypothesis that the differences between the percentages of tweets with words found for each product, and the differences of polarisation between the two analyses depend on the different marketing focus, target customer, and technological complexity of the two new video game consoles. Xbox One X targets hard-core gamers who really wants a premium experience1. With its marketing campaign, Microsoft pushed the technical supremacy of its new machine over the competitors' products, fuelling a debate about its technical features amongst the potential users.

As a result, the campaign produced a more technical social discourse that allowed us achieving better results. Instead, the new Nintendo handheld console has been developed

\footnotetext{
${ }^{1}$ http://www.businessinsider.com/why-xbox-one-X-costs-500-2017-6?IR=T (last access: 17/11/2017)
} 
targeting children and families providing a model that falls somewhere in the middle of the line of 3DS consoles2.

We initially checked our hypothesis using Google Trend to compare users' search interest about technical review of the two products during the data collection period (Figure 2). Then, we analysed the number of technical articles related to the new products published by the 25 most popular video games and technology websites in the U.S, according to the ranking of SimilarWeb, a digital marketing intelligence company which publishes insights about websites. We entered queries reported in Table 6 into Google search engine to retrieve technical article within the web domains previously identified: we obtained 1.117 articles about Xbox and only 52 about Nintendo, proving that technical debate concerning Xbox is greater. This is and evidence of the fact that when a product has a certain technological complexity and fuels a more technical debate, advantages and drawbacks analysis is more able than sentiment in producing technical-functional judgements. The greater number of neutral tweets found with advantages and drawbacks analysis can also be explained with the Means-end chain model (Reynolds, 1995). Consumers express themselves basing on personal consequences linked with product use or basing on personal values satisfied by the product itself. For these reasons, tweets contain a more colloquial language which sentiment analysis is more able to interpret than the latter tool.

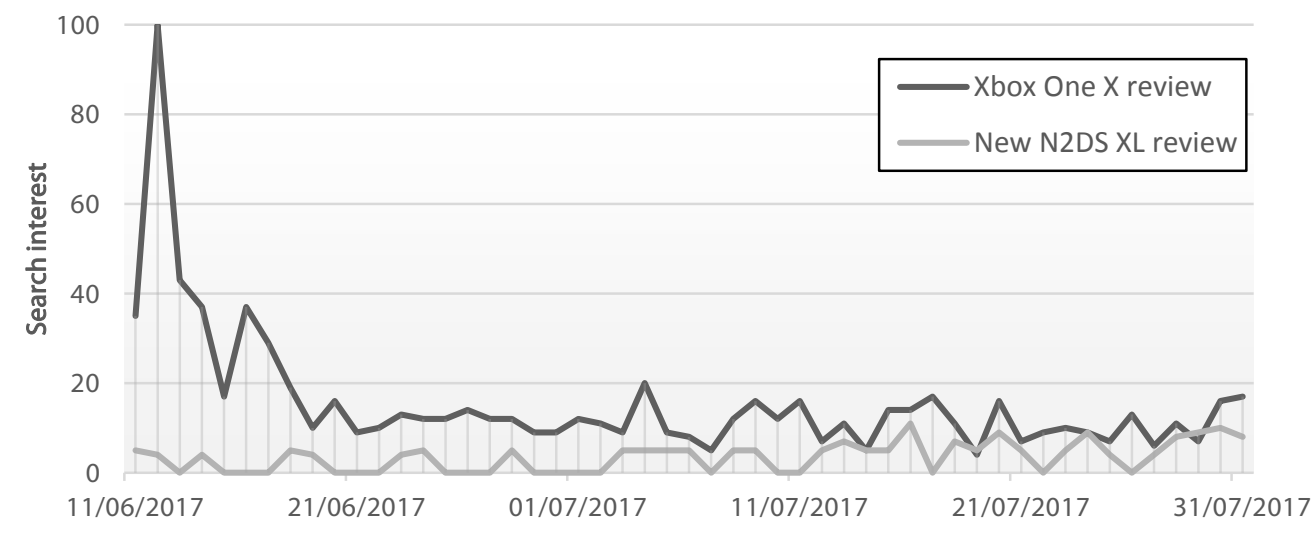

Figure 2: Google Trends comparison of search-terms "Xbox One X review" and "New Nintendo 2DS XL review" during the data collection period, since 11th June 2017 to 31st July 2017. Values on the vertical axis depict search interest compared to the highest point in the graph during the observation time. A value of 100 is the peak popularity for the term. On average, users searched for Xbox reviews with an approximately five times higher frequency.

\footnotetext{
${ }^{2}$ http://www.nintendolife.com/news/2017/05/reggie_explains_the_reasoning_behind_the_new_2ds_xl (last access: 17/11/2017)
} 
Table 6: Queries entered into Google search engine to search for technical articles within selected web domains. We selected keywords related to technical features of the products. The example report queries used for one of the analysed website: ign.com

\begin{tabular}{cl}
\hline \multirow{4}{*}{ Xbox One } & allintitle: (4k OR hdr OR hardware OR graphics OR \\
& review OR resolution OR fps OR fast OR comparison \\
& OR frame OR enhanced OR performance OR cpu OR gpu \\
& OR ram) AND ("xbox one x") site: ign.com \\
\hline \multirow{3}{*}{ New Nintendo 2DS XL } & allintitle: (graphics OR review OR screen OR \\
& comparison OR enhanced OR performance OR cpu OR \\
& gpu OR ram OR battery OR weight) AND "new nintendo \\
& 2ds xl" site: ign.com \\
\hline
\end{tabular}

\section{Conclusion}

Methods and techniques for social media mining with sentiment analysis is one of the most appreciated tool amongst researchers, having a very good reputation in the informatic fields. Also, big companies make use of it because it can be a rich source of information to adjust marketing strategies, improve campaign success, advertising message, and customer service. Nevetheless sentiment analysis is designed to extract feelings related sentiment polarity from tweets of user and not other kinds of polarity, like polarity related to technical advantages and drawbacks of products the users are experiencimg.

In this paper we shown how using a technical lexicon to analyse technical polarity of tweets is a more effective approach in giving technical-functional judgements about a product we respect to state of the art sentiment analysis techniques. It is particulartly true when a product has a certain technological complexity.

\section{References}

Ahn H., and Spangler W. S. (2014) “Sales prediction with social media analysis".SRII Global Conference. IEEE, 2014.

Asur S., and Huberman B. A. "Predicting the Future With Social Media". In IEEE/WIC/ACM International Conference on Web Intelligence and Intelligent Agent Technology. IEEE, 2010.

Bollen J., Mao H., and Zeng X. , 2015, “Twitter mood predicts the stock market”. Ref: http://arxiv.org/abs/1010.3003

Brown, Eric D., "Will Twitter Make You a Better Investor? A Look at Sentiment, User Reputation and Their Effect on the Stock Market" (2012). SAIS 2012 Proceedings. 7.

Cakra Y. E., and Trisedya B. D. "Stock price prediction using linear regression based on sentiment analysis". In 2015, International Conference on Advanced Computer Science and Information Systems (ICACSIS). IEEE, 2015. 
Chen J., Haber E., Kang R., Hsieh G., and Mahmud J. "Making use of derived personality: The case of social media ad targeting". In Proceedings of the International AAAI Conference on Web and Social Media (ICWSM), 2015.

Chiarello F., Fantoni G., Bonaccorsi A. (2017) Product description in terms of advantages and drawbacks: Exploiting patent information in novel ways. ICED 2017

Chung J., and Mustafaraj E. "Can collective sentiment expressed on twitter predict political elections?". In Twenty-Fifth AAAI Conference on Artificial Intelligence. AAAI, 2010.

Cimino A., Dell'Orletta F. (2016) "Tandem LSTM-SVM Approach for Sentiment Analysis“. In Proceedings of EVALITA '16, Evaluation of NLP and Speech Tools for Italian, 7 December, Napoli, Italy.

Collomb A. ,Costea C.,Brunie L. (2013). A Study and Comparison of Sentiment Analysis Methods for Reputation Evaluation.

Crone S. F., and Koeppel C. "Predicting exchange rates with sentiment indicators". In 2014, IEEE conference on computational intelligence for financial engineering \& economics (CIFEr). IEEE, 2014.

Kim Y., Suh B., and Lee K. "\#nowplaying the future billboard: mining music listening behaviors of twitter users for hit song prediction". In Proceedings of the first international workshop on social media retrieval and analysis, pages 51-56. ACM, 2014.

Kordonis J., Symeonidis S., and Arampatzis A. "Stock price forecasting via sentiment analysis on Twitter". In Proceedings of the 20th Pan-Hellenic Conference on Informatics, article no. 36. ACM, 2016.

Korolov R., Peabody J., Lavoie A., Das S., Magdon-Ismail M., and Wallace W. “Actions are louder than words in social media". In IEEE/ACM International Conference on Advances in Social Network Analysis and Mining. IEEE, 2015.

Le L., Ferrara E., and Flammini A. "On predictability of rare events leveraging social media: a machine learning perspective". In Proceedings of the 2015 ACM on Conference on Online Social Networks. ACM, 2015.

Li Y., Rakesh V., and Reddy C. K. "Project success prediction in crowdfunding environments". In Proceedings of the Ninth ACM International Conference on Web Search and Data Mining, pages 247-256. ACM, 2016.

Mirtalaie M.A., Hussain O.K., Chang E., Hussain F.K. (2018) Sentiment Analysis of Specific Product's Features Using Product Tree for Application in New Product Development. Lecture Notes on Data Engineering and Communications Technologies

Prasetyo N. D., and Hauff C. "Twitter-based election prediction in the developing world". In Proceedings of the 26th ACM Conference on Hypertext \& Social Media, pages 149158. ACM, 2015.

Rao T., and Srivastava S. "Analyzing stock market movements using twitter sentiment analysis. In Proceedings of the 2012 International Conference on Advances in Social Networks Analysis and Mining (ASONAM 2012), pages 119-123. IEEE Computer Society, 2012.

Razzaq M. A., Qamar A. M., and Bilal H. S. M. "Prediction and Analysis of Pakistan Election 2013 based on Sentiment Analysis". In IEEE/ACM International Conference on Advances in Social Networks Analysis and Mining (ASONAM 2014). IEEE, 2014.

Reynolds T. J., Gengler C. E. e Howard D. J (1995). . A Means-End Analysis of Brand Persuasion through Advertising, "International Journal of Research in Marketing", Vol. 12, No. 3, October, pp. 257-266. 
Sang E. T. K., and Bos J.. Predicting the 2011 Dutch senate election results with Twitter. In Proceedings of the Workshop on Semantic Analysis in Social Media, pages 53-60. ACM, 2012.

Skoric M, and Poor N. "Tweets and Votes: A Study of the 2011 Singapore General Election". IEEE, 2012.

Tuarob S., and Tucker C. S. "Fad or here to stay: predicting product market adoption and longevity using large scale, social media data". In ASME 2013 International Design Engineering Technical Conferences and Computers and Information in Engineering Conference, Volume 2B: 33rd Computers and Information in Engineering Conference Portland, Oregon, USA, August 4-7, 2013.

Tumasjan A., Sprenger T. O., Sandner P. G., and Welpe I. M. "Predicting elections with twitter: what 140 characters reveal about political sentiment". In Proceedings of the Fourth International AAAI Conference on Weblogs and Social Media. AAAI, 2010.

$\mathrm{Xu}$ F., and Keselj V. "Collective sentiment mining of microblogs in 24-hour stock price movement prediction”. In IEEE 16th conference on Business Informatics. IEEE, 2014. 